\title{
Single-Incision Laparoscopic Surgery for Colon Cancer in Patient with Situs Inversus Totalis: Report of a Case
}

\author{
Yasumitsu Hirano • Masakazu Hattori • Kenji Douden • \\ Yasuo Hashizume
}

Received: 10 March 2014 / Accepted: 9 April 2014 / Published online: 22 April 2014

(C) Association of Surgeons of India 2014

\begin{abstract}
A preliminary experience with single-incision laparoscopic colectomy for colon cancer with situs inversus totalis (SIT) is reported. An 87-year-old man was admitted because of a fecal occult blood. A colonoscopy revealed cecal cancer. An air-barium and a computed tomography contrast enema showed the right-sided descending colon and the leftsided ascending colon and cecum. The surgical procedures were as follows: First, a Lap protector was inserted through a $2.5-\mathrm{cm}$ transumbilical incision. Three $5-\mathrm{mm}$ ports were placed in the Lap protector. We successfully performed ileocolectomy with lymph node dissection by using a single-incision laparoscopic approach without any technical problems. The operative time was $125 \mathrm{~min}$, and blood loss was negligible. Postoperative follow-up did not reveal any umbilical wound complications and recurrences.
\end{abstract}

Keywords Colon cancer · Single-incision laparoscopic surgery $\cdot$ Situs inversus totalis $\cdot$ Laparoscopic colectomy

\section{Introduction}

Situs inversus totalis (SIT) is a rare congenital disorder, in 1 of 5,000 to 10,000 live births [1]. SIT denotes a complete rightleft inversion of thoracic and abdominal viscera. Accordingly, surgical procedures are considered more difficult in patients with SIT than others because of different anatomic positions of organs, especially in laparoscopic surgery. While laparoscopic colorectal surgery recently has become a standard procedure, only seven previous reports of laparoscopic

Y. Hirano $(\varangle) \cdot$ M. Hattori $\cdot$ K. Douden $\cdot$ Y. Hashizume Department of Surgery, Fukui Prefectural Hospital, 2-8-1 Yotsui, Fukui 910-8526, Japan

e-mail: yasumitsuhira@yahoo.co.jp surgery for colonic disease in patients with SIT are available [2-8].

Recently, single-incision laparoscopic colectomy (SILC) has been described through case reports and small case series. The single-incision technique results in improved cosmesis with the potential for decreased pain and fewer incisional hernias. To the best of our knowledge, this is the first case report of colon cancer associated with SIT that is successfully treated with SILC.

\section{Case Presentation}

An 87-year-old man was admitted because of a fecal occult blood with severe anemia. He had not suffered previous episodes of abdominal colicky pain and distension. There was no medical history of abdominal surgery. A colonoscopy revealed cecal tumor about $50 \mathrm{~mm}$ in size, and it was diagnosed by colon biopsy as a well-differentiated adenocarcinoma. An airbarium contrast enema showed the right-sided descending colon and the left-sided ascending colon and cecum. Computed tomography showed complete transposition of thoracic and abdominal viscera, confirming SIT (Fig. 1).

\section{Surgical Procedures}

Under general anesthesia, the patient was placed in the lithotomy position. First, a Lap protector mini (LP; Hakkou Shoji, Japan) was inserted through a $2.5-\mathrm{cm}$ transumbilical incision, which protected the wound. Next, an EZ-access (Hakkou Shoji, Japan) was mounted onto the LP, and three 5$\mathrm{mm}$ ports were made in the EZ-access. At the observation of laparoscopy, the cecum and ascending colon were situated at the left, and the sigmoid colon was situated at the right. The tumor was located at the cecum. First, the left-sided colon was mobilized using a medial approach. The ileocolic vessels were 


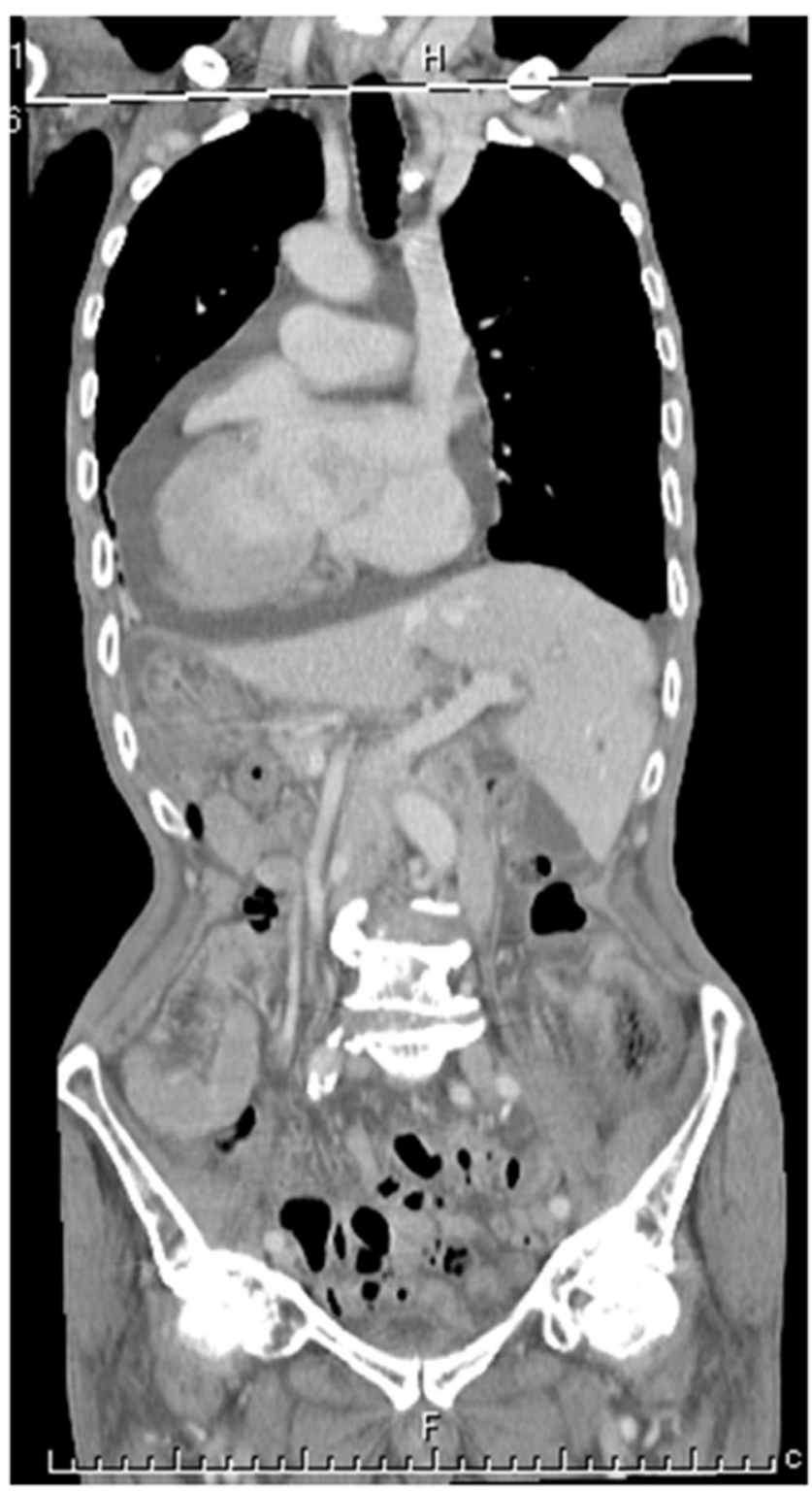

Fig. 1 Computed tomography showed a complete transposition of the thoracic and abdominal viscera

divided at their root, and all of the soft tissues anterior to the superior mesenteric vein were completely removed (D3 lymph node dissection). After mobilizing the left-sided colon, the specimen was extracted through the small incision. Resection was achieved following extracorporealization, and anastomosis was performed extracorporeally using staplers. The operative time was $125 \mathrm{~min}$, and blood loss was a negligible. The final length of skin incision was $2.5 \mathrm{~cm}$. The number of harvested lymph nodes was 25. Pathological examination revealed a well-differentiated adenocarcinoma of the cecum infiltrating the subserosal layer without nodal involvement. He received oral food on the third postoperative day. He was discharged on the 16th postoperative day. During a follow-up period of 30 months, he was free of recurrence and any umbilical wound complications (Fig. 2).

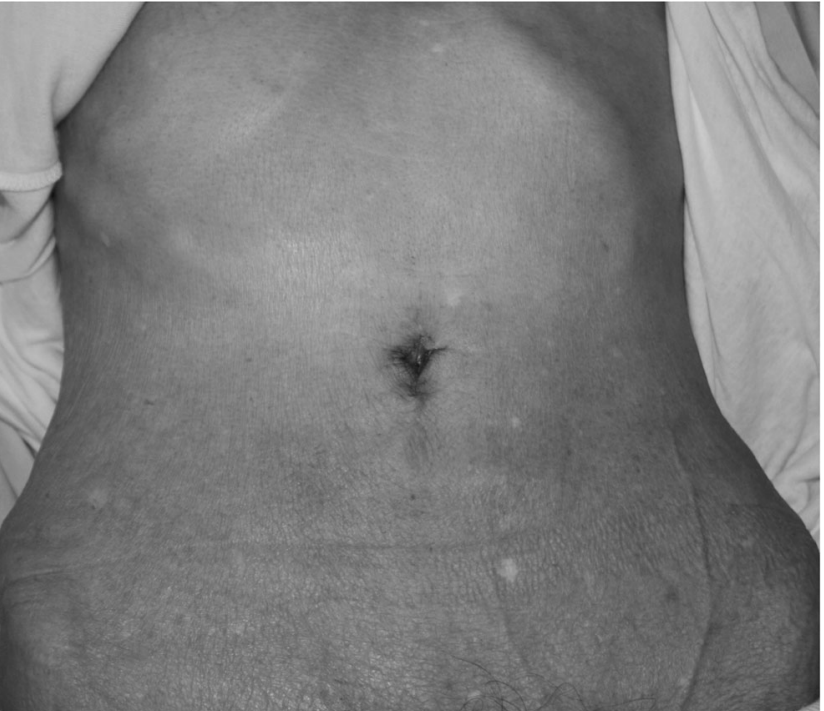

Fig. 2 Umbilical incision after 7 days

\section{Discussion}

SIT has a reported incidence of in 1 of 5,000 to 10,000 live births, making this positional anomaly very uncommon [1]. It is characterized by a transposition of the abdominal viscera, and when associated with a dextrocardia, it is referred to as situs inversus totalis. Yet, because SIT is a perfect mirror image, the relationship between the organs is not changed, so functional problems rarely occur. Generally, patients with SIT are asymptomatic and have a normal life expectancy.

Several reports on surgical procedures in SIT patients have documented greater technical difficulties, as the anatomy is abnormal. Laparoscopy can prove to be an even greater technical challenge. Although laparoscopic colorectal surgery has recently become a standard procedure, there are only seven reports on laparoscopic surgery in patients with SIT [2-8]. There are three sigmoidectomies, two hemicolectomies, one abdominoperineal resection, and one total mesenteric excision. These procedures are extremely uncommon as a result of the small patient population and a reluctance to attempt laparoscopy due to the altered anastomotic orientation.

In this case, with regard to oncologic clearance, the numbers of lymph nodes harvested were comparable, and the mean length of the resected specimen was also acceptable, with adequate tumor-free distal and proximal surgical margins. We could perform radical oncologic resection in this case with the entire regional mesocolon of the tumor and its lymphatic drainage, including a high arterial tie, and we believe that the oncological safety was maintained.

SILC is a good option for patients with SIT. Careful preoperative planning of single-incision laparoscopic procedures and careful recognition of the mirror-image anatomy permitted safe radical lymphadenectomy and mobilization of the colon, while the surgical technique itself did not differ from 
the usual situation. Clearly, the surgeon's experience with advanced SILC is a crucial factor affecting the choice of the operating method.

Our presented case demonstrated that SILC is a safe, feasible, and a curative procedure for colon cancer even in patients associated with SIT.

\section{References}

1. Nursal TZ, Baykal A, Iret D et al (2001) Laparoscopic cholecystectomy in a patient with situs inversus totalis. J Laparoendosc Adv Surg Tech A 11:239-241

2. Kim HJ, Choi GS, Park JS et al (2011) Laparoscopic right hemicolectomy with D3 lymph node dissection for a patient with situs inversus totalis: report of a case. Surg Today 41:1538-1542
3. Choi SI, Park SJ, Kang BM et al (2011) Laparoscopic abdominoperineal resection for rectal cancer in a patient with situs inversus totalis. Surg Laparosc Endosc Percutan Tech 21: e87-e90

4. Huh JW, Kim HR, Cho SH et al (2010) Laparoscopic total mesorectal excision in a rectal cancer patient with situs inversus totalis. J Korean Med Sci 25:790-793

5. Jobanputra S, Safar B, Wexner SD (2007) Laparoscopic diverticular resection with situs inversus totalis (SIT): report of a case. Surg Innov 14:284-286

6. Fujiwara Y, Fukunaga Y, Higashino M et al (2007) Laparoscopic hemicolectomy in a patient with situs inversus totalis. World $\mathrm{J}$ Gastroenterol 13:5035-5037

7. Davies H, Slater GH, Bailey M (2003) Laparascopic sigmoid colectomy for diverticular disease in a patient with situs inversus. Surg Endosc 17:160-161

8. Kobus C, Targarona EM, Bendahan GE et al (2004) Laparoscopic surgery in situs inversus: a literature review and a report of laparoscopic sigmoidectomy for diverticulitis in situs inversus. Langenbeck's Arch Surg 389:396-399 